\title{
Review of: "Regulation-based probabilistic substance quality index and automated geo-spatial modeling for water quality assessment"
}

\section{Gagan Matta}

Potential competing interests: The author(s) declared that no potential competing interests exist.

Paper is good and focused is completely based on the need of the hour, aiming for similiar kind of studies in other regions. 